\title{
A Vector Fast Multipole Algorithm for Low Frequency Problems
}

\author{
Yang G. Liu ${ }^{\# 1}$, Weng Cho Chew ${ }^{* 2}$ \\ \# Department of Electrical and Electronic Engineering, the University of Hong Kong \\ Pokfulam Road, Hong Kong, HKSAR, China \\ ${ }^{1}$ liuyang@eee.hku.hk \\ * Department of Electrical and Electronic Engineering, the University of Hong Kong, \\ Pokfulam Road, Hong Kong, HKSAR, China. \\ On Leave of Absence from the University of Illinois, Urbana-Champaign. \\ 2 wcchew@hku.hk
}

\begin{abstract}
Instead of the traditional factorization of the scalar Green's function by using scalar addition theorem in the lowfrequency fast multipole algorithm (LF-FMA), we adopt the vector addition theorem (VAT) for the factorization of the dyadic Green's function to realize memory savings for large scale problems. We validate this factorization and use it to develop a low-frequency vector fast multipole algorithm (LF-VFMA) for low-frequency problems.
\end{abstract}

\section{INTRODUCTION}

Electromagnetic simulations in the low frequency regime are important issues, where the objects or parts can be a tiny fraction of wavelength. It is necessary to improve the ability of fast solvers for handling large-scale problems at low frequencies. For achieving this aim, one way is to enhance the memory efficiency of fast solvers. To develop efficient fast solvers for low-frequency large-scale problems, we start by studying the electric field integral equation (EFIE) operator [10], which has two parts. One is due to the vector potential and the other one is due to the scalar potential. When the frequency $\omega \rightarrow 0$, the contribution from the vector potential will be lost in the numerical simulation due to finite machine precision. Then EFIE will only have the scalar potential part. The integral operator corresponding to the scalar potential part has a null space, which makes the impedance matrix nearly singular and the matrix equation difficult to solve [2], [3].

The low-frequency fast multipole algorithm (LF-FMA) has been developed to numerically solve low frequency problems. In the method, the scalar addition theorem has been used to factorize the scalar Green's function. In this paper, instead of this traditional factorization of the scalar Green's function by using scalar addition theorem, we develop a low-frequency vector fast multipole algorithm (LF-VFMA) by using the factorization of the dyadic Green's function with the vector addition theorem to accelerate the computation and save memory for far field interactions. Since vector translators of the LF-VFMA can be expressed by using scalar translators of the LF-FMA, theoretically we can only store scalar translators. However, to make the LF-VFMA numerically applicable to low-frequency problems, some elements of vector translators should be specially handled and stored. Therefore, the storage for vector translators is larger than that of scalar translators of the LF-FMA. Fortunately, the storage for vector translators is independent of the number of unknowns. Meanwhile, the storage of radiation and receiving patterns in the LF-VFMA can be reduced by 25 percent compared with that of the LF-FMA. As the storage of radiation and receiving patterns depends on the number of unknowns, it becomes the main part of the total storage with the increasing scale of problems. Hence it is effective for large scale problems to reduce the storage of radiation and receiving patterns.

\section{FACTORIZATION OF DYADIC GREEN'S FUNCTION}

In this section, we will present the factorization of the dyadic Green's function in terms of one set of vector multipole fields for developing the LF-VFMA.

A set of vector multipole fields is defined by [9]

$$
\begin{aligned}
& \mathbf{F}_{J M}(\mathbf{r})=\frac{i z_{J}(k r)}{\sqrt{J(J+1)}} \nabla_{s} Y_{J, M}(\theta, \phi) \times \mathbf{e}_{r} \\
& \mathbf{H}_{J M}(\mathbf{r})=z_{J+1}(k r)\left[\frac{\nabla_{s} Y_{J, M}-(J+1) Y_{J, M} \mathbf{e}_{r}}{\sqrt{(2 J+1)(J+1)}}\right] \\
& \mathbf{T}_{J M}(\mathbf{r})=z_{J-1}(k r)\left[\frac{J Y_{J, M} \mathbf{e}_{r}+\nabla_{s} Y_{J, M}}{\sqrt{2 J+1} \sqrt{J}}\right]
\end{aligned}
$$

where $Y_{J, M}$ is the spherical harmonics [1] $(J=0,1,2, \ldots$, and $M=-J, \ldots,-1,0,1, \ldots, J) . z_{J}(x)$ is the first kind spherical Hankel function of order $J$. Here $\mathbf{e}_{r}$ is the unit vector of $\mathbf{r}$. For this set of vector multipole fields, we let

$$
\widetilde{\widetilde{\Psi}}_{J, M}^{t}(\mathbf{r})=\left[\mathbf{F}_{J, M}(\mathbf{r}), \mathbf{H}_{J, M}(\mathbf{r}), \mathbf{T}_{J, M}(\mathbf{r})\right] \in \mathbb{C}_{3 \times 3},
$$

and then the vector addition theorem based on the set of vector multipole fields can be obtained

$$
\begin{aligned}
& \overline{\widetilde{\Psi}}_{J M}^{t}(\mathbf{r})=\sum_{J^{\prime} M^{\prime}} \mathfrak{R} g \overline{\widetilde{\Psi}}_{J^{\prime} M^{\prime}}^{t}\left(\mathbf{r}^{\prime}\right) \cdot \overline{\widetilde{\alpha}}_{J^{\prime} M^{\prime}, J M}\left(\mathbf{r}^{\prime \prime}\right),\left|\mathbf{r}^{\prime}\right|<\left|\mathbf{r}^{\prime \prime}\right| \\
& \overline{\widetilde{\Psi}}_{J M}^{t}(\mathbf{r})=\sum_{J^{\prime} M^{\prime}} \overline{\widetilde{\Psi}}_{J^{\prime} M^{\prime}}^{t}\left(\mathbf{r}^{\prime}\right) \cdot \overline{\widetilde{\beta}}_{J^{\prime} M^{\prime}, J M}\left(\mathbf{r}^{\prime \prime}\right),\left|\mathbf{r}^{\prime}\right|>\left|\mathbf{r}^{\prime \prime}\right| \\
& \mathfrak{R} g \widetilde{\boldsymbol{\Psi}}_{J M}^{t}(\mathbf{r})=\sum_{J^{\prime} M^{\prime}} \mathfrak{R} g \overline{\widetilde{\Psi}}_{J^{\prime} M^{\prime}}^{t}\left(\mathbf{r}^{\prime}\right) \cdot \overline{\widetilde{\beta}}_{J^{\prime} M^{\prime}, J M}\left(\mathbf{r}^{\prime \prime}\right),
\end{aligned}
$$


where the translator $\overline{\widetilde{\beta}}_{J^{\prime} M^{\prime}, J M}(\mathbf{r})$ is the regular part of the translator $\overline{\widetilde{\alpha}}_{J^{\prime} M^{\prime}, J M}(\mathbf{r})$.

Moreover, we have

$$
\begin{aligned}
\overline{\widetilde{\alpha}}_{J^{\prime} M^{\prime}, J M}\left(\mathbf{r}_{i l}\right) & =\sum_{J_{1}, M_{1}} \sum_{J_{2}, M_{2}} \overline{\widetilde{\beta}}_{J^{\prime} M^{\prime}, J_{1} M_{1}}\left(\mathbf{r}_{i j}\right) \\
& \cdot \overline{\widetilde{\alpha}}_{J_{1} M_{1}, J_{2} M_{2}}\left(\mathbf{r}_{j k}\right) \cdot \overline{\widetilde{\beta}}_{J_{2} M_{2}, J M}\left(\mathbf{r}_{k l}\right)
\end{aligned}
$$

In the above Equation (3), we denote the translator matrices $\overline{\widetilde{\alpha}}_{J_{1} M_{1}, J_{2} M_{2}}\left(\mathbf{r}_{j k}\right), \widetilde{\widetilde{\beta}}_{J_{2} M_{2}, J M}\left(\mathbf{r}_{k l}\right)$ and $\widetilde{\widetilde{\beta}}_{J^{\prime} M^{\prime}, J_{1} M_{1}}\left(\mathbf{r}_{i j}\right)$ as O2I translator matrix, $\mathrm{O} 2 \mathrm{O}$ translator matrix and I2I translator matrix respectively. All these translator matrices can be calculated by using $A_{J^{\prime} M^{\prime}, J M}, B_{J^{\prime} M^{\prime}, J M}$ and the scalar translators $\alpha_{J^{\prime} M^{\prime}, J M}$, which are given in [4], [9].

Moreover, for low frequencies or small structures,

$$
\begin{aligned}
& A_{J_{1} M_{1}, J_{2} M_{2}} \approx O\left(t^{-\left(J_{1}+J_{2}+1\right)}\right) \\
& B_{J_{1} M_{1}, J_{2} M_{2}} \approx O\left(t^{-\left(J_{1}+J_{2}\right)}\right) \\
& \alpha_{J_{1} M_{1}, J_{2} M_{2}} \approx O\left(t^{-\left(J_{1}+J_{2}+1\right)}\right)
\end{aligned}
$$

where $t$ is a parameter that satisfies $t / k r \approx O(1)$. Here $k$ is the wave number. By using (4), we can calculate the order of each element of the O2I translator matrix. It should be noted that the order of the $(3,3)$ element of the translator $\overline{\widetilde{\alpha}}_{J_{1} M_{1}, J_{2} M_{2}}$ is $O\left(t^{-\left(J_{1}+J_{2}-1\right)}\right)$, which is given by cancelling two highest order terms. Theoretically, these two highest order terms should cancel each other in the subtraction operation. However, for low frequency problems, the numerical error due to subtraction of highest order terms will swamp the real value of the $(3,3)$ element. Hence, we should deal with the $(3,3)$ element specially and store it alone.

In like manner, the order of each element of the $\mathrm{O} 2 \mathrm{O}$ translator $\widetilde{\widetilde{\beta}}_{J_{2} M_{2}, J M}$ and the I2I translator $\widetilde{\widetilde{\beta}}_{J^{\prime} M^{\prime}, J_{1} M_{1}}$ can be obtained. Moreover, just like the $(3,3)$ element of the O2I translator $\overline{\widetilde{\alpha}}_{J_{1} M_{1}, J_{2} M_{2}}$, the $(2,3)$ element of the $\mathrm{O} 2 \mathrm{O}$ translator $\widetilde{\widetilde{\beta}}_{J_{2} M_{2}, J M}$ and the $(3,2)$ element of the I2I translator $\widetilde{\widetilde{\beta}}_{J^{\prime} M^{\prime}, J_{1} M_{1}}$ should be calculated and stored alone to guarantee the accuracy of these elements.

After presenting the vector addition theorem based on vector multipole fields, we can factorize the dyadic Green's function with vector addition theorem as

$$
\begin{aligned}
& \overline{\mathbf{G}}\left(\mathbf{r}_{1}, \mathbf{r}_{2}\right)=\overline{\mathbf{I}} g\left(\mathbf{r}_{1}, \mathbf{r}_{2}\right)-\frac{\nabla_{1} \nabla_{2}}{k^{2}} g\left(\mathbf{r}_{1}, \mathbf{r}_{2}\right) \\
& =i k \sum_{J, M}\left[\mathbf{F}_{J, M}\left(\mathbf{r}_{14}\right) \Re g \mathbf{F}_{J, M}^{* t}\left(\mathbf{r}_{24}\right)+\mathbf{H}_{J, M}\left(\mathbf{r}_{14}\right)\right. \\
& \left.\Re g \mathbf{H}_{J, M}^{* t}\left(\mathbf{r}_{24}\right)+\mathbf{T}_{J, M}\left(\mathbf{r}_{14}\right) \mathfrak{R} g \mathbf{T}_{J, M}^{* t}\left(\mathbf{r}_{24}\right)\right] \\
& -i k \sum_{J, M} \mathbf{L}_{J, M}\left(\mathbf{r}_{14}\right) \Re g \mathbf{L}_{J, M}^{* t}\left(\mathbf{r}_{24}\right)
\end{aligned}
$$

where

$$
\mathbf{L}_{J M}\left(\mathbf{r}_{1}\right)=\frac{\sqrt{J+1}}{\sqrt{2 J+1}} \mathbf{H}_{J M}\left(\mathbf{r}_{1}\right)+\frac{\sqrt{J}}{\sqrt{2 J+1}} \mathbf{T}_{J M}\left(\mathbf{r}_{1}\right),
$$

Then, using (3) in (5), the dyadic Green's function is re- expressed as

$$
\begin{aligned}
& \overline{\mathbf{G}}\left(\mathbf{r}_{1}, \mathbf{r}_{2}\right)=i k \sum_{J, M} \sum_{J^{\prime}, M^{\prime}} \mathfrak{R} g \overline{\widetilde{\Psi}}_{J^{\prime}, M^{\prime}}^{t}\left(\mathbf{r}_{13}\right) \\
& \cdot \overline{\widetilde{\alpha}}_{J^{\prime} M^{\prime}, J M}\left(\mathbf{r}_{34}\right) \cdot \mathfrak{R} g \widetilde{\mathbf{\Psi}}_{J M}^{*}\left(\mathbf{r}_{24}\right)-i k \sum_{J, M} \sum_{J^{\prime}, M^{\prime}} \\
& \mathfrak{R} g \mathbf{L}_{J^{\prime}, M^{\prime}}\left(\mathbf{r}_{13}\right) \cdot \alpha_{J^{\prime} M^{\prime}, J M}\left(\mathbf{r}_{34}\right) \cdot \mathfrak{R} g \mathbf{L}_{J, M}^{* t}\left(\mathbf{r}_{24}\right)
\end{aligned}
$$

In addition, Equation (6) is important for saving memory in the radiation and receiving patterns of the LF-VFMA, which will be introduced in Section IV.

\section{General EQuations}

To avoid unnecessary complexity for introducing our method, we assume that the target is a perfect electric conductor. A brief review of the electric-field integral equation based on the loop-tree basis will be given in this section. Definitions of symbols in this section are similar to those in [6].

The electric field integral equation (EFIE) for an arbitrary 3D PEC object can be written as

$$
-\hat{\mathbf{t}}(\mathbf{r}) \cdot \mathbf{E}^{i n c}(\mathbf{r})=i \omega \mu \hat{\mathbf{t}}(\mathbf{r}) \cdot \int_{S} \overline{\mathbf{G}}\left(\mathbf{r}, \mathbf{r}^{\prime}\right) \cdot \mathbf{J}\left(\mathbf{r}^{\prime}\right) d \mathbf{r}^{\prime}, \mathbf{r} \in S
$$

where $\mathbf{E}^{i n c}$ is the incident electric field, $\mathbf{J}$ is the surface current, and $\hat{\mathbf{t}}(\mathbf{r})$ is an arbitrary tangential unit vector on the surface $S$. With the loop-tree basis, the basis rearrangement and frequency normalization [6], we can obtain the matrix equation of (8)

$$
\begin{aligned}
& {\left[\begin{array}{cc}
\frac{4 \overline{\mathbf{I}}}{i \omega \mu} & \overline{\mathbf{0}} \\
\overline{\mathbf{0}} & \epsilon \overline{\mathbf{K}}^{t-1}
\end{array}\right] \cdot \overline{\mathbf{F}}^{t} \cdot \overline{\mathbf{Z}}_{\mathrm{RWG}}^{V} \cdot \overline{\mathbf{F}}\left[\begin{array}{cc}
\overline{\mathbf{I}} & \overline{\mathbf{0}} \\
\overline{\mathbf{0}} & i \omega \overline{\mathbf{K}}^{-1}
\end{array}\right]} \\
& \cdot\left[\begin{array}{c}
\mathbf{I}_{L} \\
\mathbf{Q}
\end{array}\right]+\left[\begin{array}{cc}
\overline{\mathbf{0}} & \overline{\mathbf{0}} \\
\overline{\mathbf{0}} & i \omega \epsilon \overline{\mathbf{K}}^{t-1} \cdot \overline{\mathbf{Z}}_{C C}^{S} \cdot \overline{\mathbf{K}}^{-1}
\end{array}\right] \cdot\left[\begin{array}{c}
\mathbf{I}_{L} \\
\mathbf{Q}
\end{array}\right] \\
& =\left[\begin{array}{c}
\frac{4 \mathbf{V}_{L}}{i \omega \mu} \\
\epsilon \mathbf{K}^{t-1} \cdot \mathbf{V}_{C}
\end{array}\right]
\end{aligned}
$$

where the element of the matrix $\overline{\mathbf{Z}}_{\mathrm{RWG}}^{V}$ is

$$
\left[\overline{\mathbf{Z}}_{\mathrm{RWG}}^{V}\right]_{m, n}=i \omega \mu\left\langle\mathbf{J}_{R_{m}}(\mathbf{r}), \overline{\mathbf{I}} g\left(\mathbf{r}, \mathbf{r}^{\prime}\right), \mathbf{J}_{R_{n}}^{t}\left(\mathbf{r}^{\prime}\right)\right\rangle,
$$

where $m, n=1, \cdots, N_{R} . \mathbf{J}_{R_{m}}$ is the $m$-th RWG basis and $N_{R}$ is the number of RWG basis. The matrix $\overline{\mathbf{F}}^{t}$ is a transformation matrix that changes from the RWG basis to the loop-tree basis. The matrix $\overline{\mathbf{K}}$ corresponds to the process of the basis rearrangement with the relationship

$$
\overline{\mathbf{K}} \cdot \mathbf{I}_{C}=i \omega \mathbf{Q} .
$$

\section{LF-VFMA BASED ON THE VAT}

Based on formulations introduced in the last section, we will present the LF-VFMA in this section. The storage requirement for radiation and receiving patterns and vector translators is discussed to show the memory efficiency of this method for large scale problems.

We will introduce the low-frequency vector fast multipole algorithm in two steps. First we consider the matrix-vector 
multiplication in the first term on the left hand side of (9). The fast multipole algorithm is used to accelerate the multiplication of the matrix $\overline{\mathbf{Z}}_{\mathrm{RWG}}^{V}$ and a vector. Any element $\left[\overline{\mathbf{Z}}_{\mathrm{RWG}}^{V}\right]_{m, n}$ can be expressed as

$$
\begin{aligned}
& -\omega \mu k \sum_{J, M} \sum_{J^{\prime}, M^{\prime}}\left\langle\mathbf{J}_{R_{m}}\left(\mathbf{r}_{1}\right), \mathfrak{R} g \overline{\widetilde{\Psi}}_{J^{\prime}, M^{\prime}}^{t}\left(\mathbf{r}_{13}\right)\right\rangle \\
& \overline{\widetilde{\alpha}}_{J^{\prime} M^{\prime}, J M}\left(\mathbf{r}_{34}\right) \cdot\left\langle\mathfrak{R} g \overline{\widetilde{\Psi}}_{J, M}^{*}\left(\mathbf{r}_{24}\right), \mathbf{J}_{R_{n}}^{t}\left(\mathbf{r}_{2}\right)\right\rangle
\end{aligned}
$$

where the receiving and radiation patterns are given as follows respectively.

$$
\begin{aligned}
& \left\langle\mathbf{J}_{R_{m}}\left(\mathbf{r}_{1}\right), \mathfrak{R} g \overline{\widetilde{\Psi}}_{J^{\prime}, M^{\prime}}^{t}\left(\mathbf{r}_{13}\right)\right\rangle \\
& =\left[\left\langle\mathbf{J}_{R_{m}}\left(\mathbf{r}_{1}\right), \mathfrak{R} g \mathbf{F}_{J^{\prime} M^{\prime}}\left(\mathbf{r}_{13}\right)\right\rangle,\left\langle\mathbf{J}_{R_{m}}\left(\mathbf{r}_{1}\right),\right.\right. \\
& \left.\left.\Re \mathfrak{R} \mathbf{H}_{J^{\prime} M^{\prime}}\left(\mathbf{r}_{13}\right)\right\rangle,\left\langle\mathbf{J}_{R_{m}}\left(\mathbf{r}_{1}\right), \mathfrak{R} g \mathbf{T}_{J^{\prime} M^{\prime}}\left(\mathbf{r}_{13}\right)\right\rangle\right] \\
& \left\langle\mathfrak{R} g \widetilde{\boldsymbol{\Psi}}_{J, M}^{*}\left(\mathbf{r}_{24}\right), \mathbf{J}_{R_{n}}^{t}\left(\mathbf{r}_{2}\right)\right\rangle \\
& =\left[\left\langle\mathfrak{R} g \mathbf{F}_{J, M}^{* t}\left(\mathbf{r}_{24}\right), \mathbf{J}_{R_{n}}^{t}\left(\mathbf{r}_{2}\right)\right\rangle,\left\langle\mathfrak{R} g \mathbf{H}_{J, M}^{* t}\left(\mathbf{r}_{24}\right),\right.\right. \\
& \left.\left.\mathbf{J}_{R_{n}}^{t}\left(\mathbf{r}_{2}\right)\right\rangle,\left\langle\mathfrak{R} g \mathbf{T}_{J, M}^{* t}\left(\mathbf{r}_{24}\right), \mathbf{J}_{R_{n}}^{t}\left(\mathbf{r}_{2}\right)\right\rangle\right]^{t}
\end{aligned}
$$

After obtaining the factorization of the elements of the matrix $\overline{\mathbf{Z}}_{\mathrm{RWG}}^{V}$, the implementation process of the multiplication of the matrix $\overline{\mathbf{Z}}_{\mathrm{RWG}}^{V}$ and a vector is similar to the traditional LFFMA [7]. Then we present the scalar potential part with LFVFMA. In this part, the main calculation is the multiplication of the matrix $\overline{\mathbf{Z}}_{C C}^{S}$ and a vector. We can expand the element in the far field part of the matrix $\overline{\mathbf{Z}}_{C C}^{S}$ as

$$
\begin{aligned}
& {\left[\overline{\mathbf{Z}}_{C C}^{S}\right]_{m, n}=\frac{k^{3}}{\omega \epsilon}\left\langle\mathbf{J}_{C_{m}}\left(\mathbf{r}_{1}\right), \frac{\nabla \nabla^{\prime}}{k^{2}} g\left(\mathbf{r}_{1}, \mathbf{r}_{2}\right), \mathbf{J}_{C_{n}}^{t}\left(\mathbf{r}_{2}\right)\right\rangle} \\
& =\frac{k^{3}}{\omega \epsilon} \sum_{J M} \sum_{J^{\prime} M^{\prime}}\left\langle\mathbf{J}_{C_{m}}\left(\mathbf{r}_{1}\right), \mathfrak{R} g \mathbf{L}_{J^{\prime} M^{\prime}}\left(\mathbf{r}_{13}\right)\right\rangle \\
& \cdot \alpha_{J^{\prime} M^{\prime}, J M}\left(\mathbf{r}_{34}\right) \cdot\left\langle\mathfrak{R} g \mathbf{L}_{J, M}^{* t}\left(\mathbf{r}_{24}\right), \mathbf{J}_{C_{n}}^{t}\left(\mathbf{r}_{2}\right)\right\rangle
\end{aligned}
$$

where $\mathbf{J}_{C_{m}}$ is the $m$-th tree basis. If we adopt (15) to calculate the matrix $\overline{\mathbf{Z}}_{C C}^{S}$ in the scalar potential part of low-frequency large-scale problems, the subtraction error accumulated in the basis rearrangement process will lead to the divergence of the iteration. To eliminate the subtraction error, we can use the patch-pair basis to express charges [8].

By using patch-pair basis, the term on the left hand side of (9) can be rewritten as

$$
i \omega \epsilon \overline{\mathbf{K}}^{t-1} \cdot \overline{\mathbf{Z}}_{C C}^{S} \cdot \overline{\mathbf{K}}^{-1} \mathbf{Q}=i \omega \epsilon \overline{\mathbf{W}}^{t} \cdot \overline{\mathbf{Z}}_{P P}^{S} \cdot \overline{\mathbf{W}} \cdot \mathbf{Q}
$$

where the matrix $\overline{\mathbf{W}}$ is a transformation matrix. Here, $N_{P}$ is the number of single patches, $N_{C}$ is the number of tree basis and $N_{P}=N_{C}+1$. Moreover, $\overline{\mathbf{Z}}_{P P}^{S}$ is a $N_{P} \times N_{P}$ matrix, whose element in the far field part can be written as

$$
\begin{aligned}
& {\left[\overline{\mathbf{Z}}_{P P}^{S}\right]_{m n}=-\frac{i}{\omega \epsilon}\left\langle J_{P_{m}}\left(\mathbf{r}_{1}\right), g\left(\mathbf{r}_{1}, \mathbf{r}_{2}\right), J_{P_{n}}\left(\mathbf{r}_{2}\right)\right\rangle} \\
& =\frac{k}{\omega \epsilon} \sum_{J, M} \sum_{J^{\prime}, M^{\prime}}\left\langle J_{P_{m}}\left(\mathbf{r}_{1}\right), \Re g \psi_{J^{\prime}, M^{\prime}}\left(\mathbf{r}_{1}-\mathbf{r}_{l^{\prime}}\right)\right\rangle \cdot \\
& \cdot \alpha_{J^{\prime} M^{\prime}, J M}\left(\mathbf{r}_{l^{\prime}}-\mathbf{r}_{l}\right)\left\langle\mathfrak{R} g \psi_{J M}^{*}\left(\mathbf{r}_{2}-\mathbf{r}_{l}\right), J_{P_{n}}\left(\mathbf{r}_{2}\right)\right\rangle
\end{aligned}
$$

where $\mathbf{r}_{l^{\prime}}$ denotes the center of the box to which the single patch basis function $J_{P_{m}}$ belongs.

From (16) and (17), we see that the main calculation in scalar potential part of the LF-VFMA has been converted to the multiplication of the matrix $\overline{\mathbf{Z}}_{P P}^{S}$ and a vector. By doing so, the accumulation of subtraction error can be avoided.

In the following we will show the memory saving in the part of radiation and receiving patterns of the LF-VFMA. Since radiation and receiving patterns are similar, we only consider receiving patterns in the following. By using the integration by parts, receiving patterns in (15) can be calculated by using the single patch basis

$$
\begin{aligned}
& \left\langle\mathbf{J}_{C_{m}}\left(\mathbf{r}_{1}\right), \mathfrak{R} g \mathbf{L}_{J^{\prime}, M^{\prime}}\left(\mathbf{r}_{1}-\mathbf{r}_{l}\right)\right\rangle \\
& =-\frac{1}{k}\left\langle\nabla \cdot \mathbf{J}_{C_{m}}\left(\mathbf{r}_{1}\right), \mathfrak{R} g \psi_{J^{\prime}, M^{\prime}}\left(\mathbf{r}_{1}-\mathbf{r}_{l}\right)\right\rangle \\
& =-\frac{1}{k}\left(\left\langle J_{P_{t_{1}}}\left(\mathbf{r}_{1}\right), \mathfrak{R} g \psi_{J^{\prime}, M^{\prime}}\left(\mathbf{r}_{1}-\mathbf{r}_{l}\right)\right\rangle\right. \\
& \left.-\left\langle J_{P_{t_{2}}}\left(\mathbf{r}_{1}\right), \mathfrak{R} g \psi_{J^{\prime}, M^{\prime}}\left(\mathbf{r}_{1}-\mathbf{r}_{l}\right)\right\rangle\right)
\end{aligned}
$$

where $\mathbf{r}_{l}$ is the center of the box to which the RWG basis $\mathbf{J}_{C_{m}}$ belongs. Let the center of the box to which the patch basis $J_{P_{t_{i}}}$ belongs be $\mathbf{r}_{t_{i}}, i=1,2$. It should be noted that $\mathbf{r}_{t_{1}}$ or $\mathbf{r}_{t_{2}}$ is not always equal to $\mathbf{r}_{l}$ [7]. If $\mathbf{r}_{t_{1}} \neq \mathbf{r}_{l}$, a translator is needed to transform $\left\langle J_{P_{t_{1}}}\left(\mathbf{r}_{1}\right), \Re g \psi_{J^{\prime}, M^{\prime}}\left(\mathbf{r}_{1}-\mathbf{r}_{t_{1}}\right)\right\rangle$ to $\left\langle J_{P_{t_{1}}}\left(\mathbf{r}_{1}\right), \mathfrak{R} g \psi_{J^{\prime}, M^{\prime}}\left(\mathbf{r}_{1}-\mathbf{r}_{l}\right)\right\rangle$.

By using the scalar addition theorem [7], we know

$$
\mathfrak{R} g \psi_{J, M}(\mathbf{r})=\sum_{J^{\prime}, M^{\prime}} \mathfrak{R} g \psi_{J^{\prime}, M^{\prime}}\left(\mathbf{r}^{\prime}\right) \cdot \beta_{J^{\prime} M^{\prime}, J M}\left(\mathbf{r}^{\prime \prime}\right),
$$

where $\mathbf{r}=\mathbf{r}^{\prime}+\mathbf{r}^{\prime \prime}$. So it can be obtained that

$$
\begin{aligned}
& \left\langle J_{P_{t_{1}}}\left(\mathbf{r}_{1}\right), \mathfrak{R} g \psi_{J^{\prime} M^{\prime}}\left(\mathbf{r}_{1}-\mathbf{r}_{l}\right)\right\rangle=\sum_{J, M}\left\langle J_{P_{t_{1}}}\left(\mathbf{r}_{1}\right),\right. \\
& \left.\Re g \psi_{J M}\left(\mathbf{r}_{1}-\mathbf{r}_{t_{1}}\right)\right\rangle \cdot \beta_{J M, J^{\prime} M^{\prime}}\left(\mathbf{r}_{t_{1}}-\mathbf{r}_{l}\right)
\end{aligned}
$$

Since the box to which a patch triangle of an RWG basis belongs is adjacent to or the same as the box to which the RWG basis belongs, the vector $\mathbf{r}_{t_{1}}-\mathbf{r}_{l}$ only has 27 possibilities at most. So the storage of translators $\beta_{J^{\prime} M^{\prime}, J M}$ is small and remains constant.

In addition, by applying (6), we can express the third component of receiving patterns (13) in the vector potential part of the LF-VFMA as

$$
\begin{aligned}
& \left\langle\mathbf{J}_{R_{i}}\left(\mathbf{r}_{1}\right), \mathfrak{R} g \mathbf{T}_{J M}\left(\mathbf{r}_{1}-\mathbf{r}_{l_{i}}\right)\right\rangle \\
& =\frac{\sqrt{2 J+1}}{\sqrt{J}}\left\langle\mathbf{J}_{R_{i}}\left(\mathbf{r}_{1}\right), \mathfrak{R} g \mathbf{L}_{J M}\left(\mathbf{r}_{1}-\mathbf{r}_{l_{i}}\right)\right\rangle \\
& -\frac{\sqrt{J+1}}{\sqrt{J}}\left\langle\mathbf{J}_{R_{i}}\left(\mathbf{r}_{1}\right), \mathfrak{R} g \mathbf{H}_{J M}\left(\mathbf{r}_{1}-\mathbf{r}_{l_{i}}\right)\right\rangle
\end{aligned}
$$

Since the subtraction on the right hand side of (21) is between two terms with different highest orders, the real value of $\left\langle\mathbf{J}_{R_{i}}\left(\mathbf{r}_{1}\right), \mathfrak{R} g \mathbf{T}_{J M}\left(\mathbf{r}_{1}-\mathbf{r}_{l_{i}}\right)\right\rangle$ will not be swamped by the subtraction error.

Therefore, in the scalar potential part of the LF-VFMA, we only store receiving patterns $\left\langle J_{P_{m}}\left(\mathbf{r}_{1}\right), \mathfrak{R} g \psi_{J^{\prime}, M^{\prime}}\left(\mathbf{r}_{1}-\mathbf{r}_{l^{\prime}}\right)\right\rangle$ with the single patch basis. For receiving patterns (13) in the vector potential part of the LF-VFMA, only the first 
two components need to be calculated and stored. By using (18), (20) and (21), the third component can be obtained with the second component of receiving patterns in the vector potential part, receiving patterns of the scalar potential part and translators introduced in (19). Hence, the total storage of receiving patterns contains three parts: One is receiving patterns with the single patch basis in the scalar potential part and the other two are the first two components of receiving patterns in the vector potential part. Therefore, the total storage of radiation and receiving patterns of the LF-VFMA can be reduced by 25 percent compared with that of the LF-FMA.

As for the storage for vector translators, since elements of vector translators can be calculated with scalar translators of the LF-FMA, theoretically, we can only store scalar translators for generating vector translators. However, as we have discussed in Section 2, some elements of vector translators should be calculated and stored alone to avoid numerical error due to the subtraction of two high order terms. Therefore, the storage for vector translators is larger than that for scalar translators. In addition, we should store translators used in (20), whose storage is small and remains constant.

Fortunately, the storage for all translators used in this method is independent of the number of unknowns. With the increase in the number of unknowns, the storage of radiation and receiving patterns, which depends on the number of unknowns, will dominate over that of translators. It is reduced by 25 percent in our method compared with that in the LFFMA. The analysis of memory requirement implies that the method is superior in saving memory for solving large scale problems.

\section{NUMERICAL EXAMPLES}

This section presents an almond scattering example to demonstrate the validity of the LF-VFMA.

We use an $x$-polarized plane wave incident from the $z$ direction to excite a PEC NASA almond given in Fig. 1, whose length $d=2.52374 \mathrm{~m}$. The mesh has 5,574 inner edges. We use the 6 level LF-VFMA to calculate the bistatic RCS of the PEC Nasa almond with fixed $\phi=0$ at $10^{-6} \mathrm{GHz}$. The GMRES-50 is used to solve the matrix equation. The result of the LF-VFMA is compared with that of the method of moments (MOM) in Fig. 2.

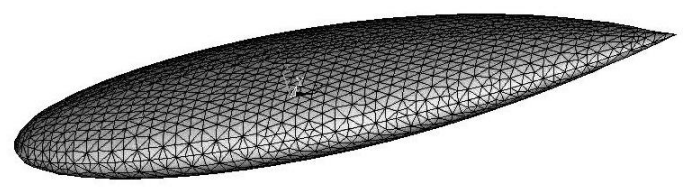

Fig. 1. NASA Almond.

\section{CONCLUSIONS}

In this work, the vector addition theorem is adopted for the factorization of the dyadic Green's function. Then the factorization is used to develop the LF-VFMA for realizing

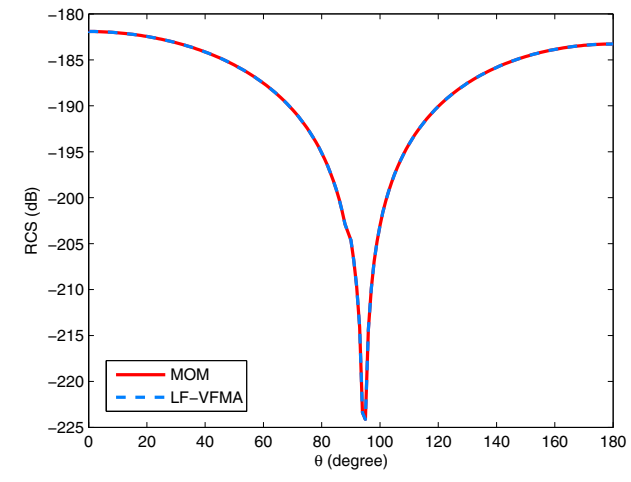

Fig. 2. Bistatic RCS of a PEC NASA almond.

memory savings. In this method, the storage of translators is larger than that of the LF-FMA. Although there is an increase in the storage of translators, it is independent of the number of unknowns. Meanwhile, the storage of radiation and receiving patterns in the far field part can be reduced by 25 percent compared with that of the LF-FMA. Since the storage of radiation and receiving patterns depends on the number of unknowns, it will become the main part of the total storage with the increase in the scale of problems. So the reduction of the storage of radiation and receiving patterns is meaningful for large scale problems.

\section{REFERENCES}

[1] J. A. Stratton, Electromagnetic Theory. New York: McGrawHill, 1941.

[2] D. R. Wilton and A. W. Glisson, "On improving the electric field integral equation at low frequencies," In Proc. URSI Radio Sci. Meet. Dig., Los Angeles, CA, June 1981, P. 24.

[3] J. R. Mautz and R. F. Harrington, "An E-field solution for a conducting surface small or comparable to the wavelength," IEEE Trans. Antennas Propagat., vol. 32, pp. 330-339, Apr. 1984.

[4] W. C. Chew, Waves and Fields in Inhomogeneous Media. New York: IEEE Press, 1995.

[5] G. Dassios and Z. Rigou, "Elastic Herglotz functions," SIAM J. Appl. Math., vol. 55, no. 5, pp. 1345-1361, 1995.

[6] J. S. Zhao and W. C. Chew, "Integral equation Solution of Maxwell's equations from zero frequency to microwave frequencies," IEEE transactions on antennas and propagation, vol. 48, no. 10, pp. 1635-1645, Oct. 2000.

[7] W. C. Chew, J. M. Jin, E. Midielssen, and J. M. Song, Fast and Efficient Algorithms in Computational Electromagnetics. Boston, MA: Artech House, 2001.

[8] Y. H. Chu and W. C. Chew, "Large-Scale Computation For Electrically Small Structures Using Surface-Integral Equation Method," Micro. Opt. Tech. Lett., vol. 47, no. 6, pp. 525-530, Dec 2005.

[9] B. He and W. C. Chew, "Diagonalizations of vector and tensor addition theorems," Commun. Comput. Phys., vol. 4, no. 4, pp. 797-819, 2008.

[10] W. C. Chew, M. S. Tong, and B. Hu, Integral Equation Methods for the Electromagnetic and Elastic Waves. Morgan and Claypool Publishers, 2009. 